\title{
Simulation of the single-vibronic-level emission spectra of HAsO and DAsO
}

\author{
Daniel K. W. Mok ${ }^{1, a)}$ Edmond P. F. Lee ${ }^{1,2, a)}$ and John M. Dyke ${ }^{2}$ \\ ${ }^{1}$ Department of Applied Biology and Chemical Technology, the Hong Kong Polytechnic University, \\ Hung Hom, Hong Kong \\ ${ }^{2}$ School of Chemistry, University of Southampton, Highfield, Southampton SO17 1BJ, UK
}

\begin{abstract}
The single-vibronic-level emission spectra of HAsO and DAsO have been simulated by electronic structure/Franck-Condon factor calculations to confirm the spectral molecular carrier and to investigate the electronic states involved. Various multi-reference (MR) methods, namely NEVPT2 (n-electron valence state second order perturbation theory), RSPT2-F12 (explicitly correlated Rayleigh-Schrodinger second order perturbation theory) and MRCI-F12 (explicitly correlated multi-reference configuration interaction), were employed to compute the geometries and relative electronic energies for the $\widetilde{\mathrm{X}}^{1} \mathrm{~A}^{\prime}$ and $\widetilde{\mathrm{A}}^{1} \mathrm{~A}^{\prime \prime}$ states of HAsO. These are the highest level calculations on these states yet reported. The MRCI-F12 method gives computed $\mathrm{T}_{0}$ (adiabatic transition energy including zero-point energy correction) values which agree well with the available experimental $\mathrm{T}_{0}$ value, much better than previously computed values and values computed with other MR methods in this work. In addition, the potential energy surfaces of the $\widetilde{\mathrm{X}}^{1} \mathrm{~A}^{\prime}$ and $\widetilde{\mathrm{A}}^{1} \mathrm{~A}^{\prime \prime}$ states of HAsO were computed using the MRCI-F12 method. Franck-Condon factors between the two states, which include anharmonicity and Duschinsky rotation, were then computed and used to simulate the recently reported single-vibronic-level (SVL) emission spectra of HAsO and DAsO [Grimminger and Clouthier, J. Chem. Phys. 135, 184308 (2011)]. Our simulated SVL emission spectra confirm the assignments of the molecular carrier, electronic states involved and the vibrational structures observed in the SVL emission spectra, but suggest a loss of intensity in the reported experimental spectra at the low emission energy region, almost certainly due to a loss of responsivity near the cut off region $(\sim 800 \mathrm{~nm})$ of the detector used. Computed and experimentally
\end{abstract}


derived $r_{e}$ (equilibrium) and/or $r_{0}$ \{the $(0,0,0)$ vibrational level $\}$ geometries of the two states of $\mathrm{HAsO}$ are discussed.

a) Authors to whom correspondence should be addressed. Electronic addresses: bcdaniel@polyu.edu.hk and epl@soton.ac.uk 


\section{Introduction}

Recently, Grimminger and Clouthier reported the $\widetilde{\mathrm{X}}^{1} \mathrm{~A}^{\prime}-\widetilde{\mathrm{A}}^{1} \mathrm{~A}^{\prime \prime}$ laser-induced fluorescence (LIF) and single vibronic level (SVL) emission spectra of HAsO and DAsO for the first time. ${ }^{1}$ In order to assist assignments of the observed spectra, density functional theory (DFT) calculations, employing the B3LYP functional, and $a b$ initio (or wavefunction) calculations, employing the coupled-cluster single-double plus perturbative triples $\{\operatorname{CCSD}(\mathrm{T})\}$ method, were carried out on the two electronic states involved, using augmented correlation-consistent valence-polarized basis sets of up to quintuple-zeta quality (aug-cc-pV5Z). Although computed harmonic vibrational frequencies of the two states reported in reference 1 agree reasonably well with available experimental fundamental vibrational frequencies measured in the LIF and SVL emission spectra (vide infra), the computed $\mathrm{T}_{0}$ values of 11731 and $14225 \mathrm{~cm}^{-1}$ (1.454 and $1.764 \mathrm{eV}$ ) obtained at the B3LYP/aug-cc-pV5Z and CCSD(T)/aug-cc-pV5Z levels of theory, respectively, are smaller than the LIF experimental value of $15316.7 \mathrm{~cm}^{-1}(1.899 \mathrm{eV})$ by $\sim 3600$ and $\sim 1100 \mathrm{~cm}^{-1}(\sim 0.445$ and $\sim 0.135 \mathrm{eV}$ ). These discrepancies between theory and experiment in the relative energies of the two electronic states of $\mathrm{HAsO}$ are significantly larger than the commonly accepted chemical accuracy of $1 \mathrm{kcal} . \mathrm{mol}^{-1}\left(\sim 0.04 \mathrm{eV}\right.$ or $\left.\sim 350 \mathrm{~cm}^{-1}\right)$. In this connection, we propose to carry out higher level calculations on the two states of $\mathrm{HAsO}$ in order to resolve the difference between theory and experiment in the relative electronic energies of the $\widetilde{\mathrm{X}}^{1} \mathrm{~A}^{\prime}-\widetilde{\mathrm{A}}^{1} \mathrm{~A}^{\prime \prime}$ transition. Specifically, multireference (MR) methods have been employed in the present study, because the excited $\widetilde{\mathrm{A}}^{1} \mathrm{~A}^{\prime \prime}$ state is an open-shell singlet state, for which single-reference (SR) methods $\{$ e.g. B3LYP and $\operatorname{CCSD}(\mathrm{T})\}$ are inadequate. In addition, following our on-going ab initio/Franck-Condon factor (FCF) research program, ${ }^{2,3}$ which has successfully provided "fingerprint" type assignments for the SVL emission spectra of a large number of triatomic molecules, ${ }^{4,5,6,7,8,9,10,11,12}$ including HPO/DPO ${ }^{13}$ and HPS/DPS,${ }^{14}$ which are valence iso-electronic with $\mathrm{HAsO} / \mathrm{DAsO}$, FCFs between the two states of $\mathrm{HAsO}$, which include allowance for anharmonicity and Duschinsky rotation, have also been computed and used to simulate the SVL emission spectra of HAsO and DAsO. ${ }^{1}$ 
Arsenic containing small molecules are of interest in chemical vapor deposition (CVD) processes in the semiconductor industry (see reference 1and references therein). Prior to reference 1, Hartree-Fock (HF) calculations on the geometry and frequencies of HAsO using polarized valence double-zeta quality basis sets were reported in $1990,{ }^{15}$ and a vibrational band at $1931 \mathrm{~cm}^{-1}$ was tentatively assigned to the H-As stretching mode of HAsO in an infrared (IR) argon matrix study of codeposition of $\mathrm{AsH}_{3}$ and $\mathrm{O}_{3}$, followed by photolysis in 1989. ${ }^{16}$ However, little previous work has been performed on HAsO. The objectives of this work were to simulate the $\widetilde{\mathrm{A}}-\widetilde{\mathrm{X}} \mathrm{SVL}$ emission spectrum of $\mathrm{HAsO}$, confirm the spectral carrier, investigate the states involved and obtain an improved computed $\mathrm{T}_{0}$ value.

\section{Computational strategy and details}

\section{Geometry optimization calculations}

All calculations were performed using the MOLPRO suite of programs. ${ }^{17,18}$ Geometry optimization calculations were carried out on the $\widetilde{\mathrm{X}}^{1} \mathrm{~A}^{\prime}$ and $\widetilde{\mathrm{A}}^{1} \mathrm{~A}^{\prime \prime}$ states of HAsO. The completeactive-space self-consistent-field (CASSCF) method, ${ }^{19}$ with a full valence active space, followed by the post-CASSCF multi-reference (MR) NEVPT2, RSPT2-F12 and MRCI-F12 methods, as implemented in MOLPRO, were employed. For the closed-shell $\widetilde{X}^{1} \mathrm{~A}^{\prime}$ state, $\operatorname{CCSD}\left(\mathrm{T}^{*}\right)-\mathrm{F} 12 \mathrm{x}(\mathrm{x}=\mathrm{a}$ or b) calculations were also performed (geometry optimization based on the F12b energies). NEVPT2, the acronym for n-electron valence state perturbation theory, is a second order MR perturbation theory method. ${ }^{20}$ One main advantage of the NEVPT2 method is the absence of intruder states. RSPT2-F12, ${ }^{21}$ MRCI-F12 ${ }^{22}$ and $\mathrm{CCSD}\left(\mathrm{T}^{*}\right)-\mathrm{F} 12^{23}$ are explicit correlation (F12) methods based on the conventional MR Rayleigh-Schrodinger second order perturbation theory (RSPT2), multi-reference configuration interaction (MRCI) and CCSD(T) methods, respectively. For the $\operatorname{CCSD}\left(\mathrm{T}^{*}\right)-\mathrm{F} 12$ method, the scaled perturbative triples $\left(\mathrm{T}^{*}\right)$ obtained by a simple scaling factor, $\Delta \mathrm{E}\left(\mathrm{T}^{*}\right)=\Delta \mathrm{E}(\mathrm{T}) \times \mathrm{E}_{\text {corr }}^{\mathrm{MP} 2-\mathrm{F} 12} / \mathrm{E}_{\text {corr }}^{\mathrm{MP} 2}$, the ratio between the computed correlation energies obtained at the RMP2 and RMP2-F12 levels, ${ }^{17,23}$ were computed and used throughout. Since 
explicitly correlated methods are known to accelerate convergence of computed correlation energies toward the complete basis set limit, ${ }^{24}$ using a small basis set (e.g. TZ quality) with these F12 methods is expected to produce results comparable to using a large basis set (e.g. 5Z quality) with the conventional counterparts $\{$ i.e. $\mathrm{RSPT} 2, \mathrm{MRCI}$ and $\operatorname{CCSD}(\mathrm{T})\} .^{25}$

The basis sets ${ }^{26,27,28,29,30,31,32}$ employed in the present study for various frozen core (FC) F12 calculations are summarized in Table 1 (see also footnotes). Most of these basis sets were designed for the F12 methods. For NEVPT2 calculations, the aug-cc-pVQZ basis sets ${ }^{33}$ for $\mathrm{O}$ and $\mathrm{H}$, and the ECP10MDF effective core potential (ECP) and associated aug-cc-pVQZ-PP basis set ${ }^{34}$ for As were used. In addition to the frozen core (FC) calculations described above, the As $3 \mathrm{~d}^{10}$ core electrons were also correlated explicitly in some MRCI-F12 calculations (core,4,1; frozen As $3 \mathrm{~s}^{2} 3 \mathrm{p}^{6}$ and O $1 s^{2}$ with As $1 s^{2} 2 p^{2} 2 p^{6}$ accounted for by the ECP10MDF ECP). In order for the As $3 d^{10}$ electrons to be correlated adequately, uncontracted tight 4d (exponents: 12.0, 4.0, 1.3333333, 0.444444), $2 \mathrm{f}$ $(14.0,6.0)$ and $2 \mathrm{~g}(9.6,2.4)$ functions were added to the atomic orbital (AO) basis set of As described in Table 1, giving in total 278 contracted basis functions for HAsO (denoted simply as CVQZ-F12 from here onward). However, in order for the CASSCF/MRCI-F12 calculations to be tractable, the FC CASSCF full valence molecular orbitals were used in the subsequent MRCI-F12 calculations, which had the As $3 \mathrm{~d}^{10}$ electrons active. In these MRCI-F12 calculations, which correlated also the As $3 \mathrm{~d}^{10}$ electrons, the geminal Slater exponent $\{\beta$, in the nonlinear correlation factor, $\left.\hat{\mathrm{F}}\left(\mathrm{r}_{12}\right)=-(1 / \beta) \exp \left(-\beta \mathrm{r}_{12}\right)\right\}$ was set to 1.5 instead of the default value of 1.0 used for valence only calculations, as recommended for core-valence F12 calculations. ${ }^{35}$ With the default frozen core, the total numbers of contracted and uncontracted configurations used in the MRCI-F12 calculations are 1516432 and 138334504 , respectively. With the As $3 \mathrm{~d}^{10}$ electrons also active in the MRCI-F12 calculations, the numbers of contracted and uncontracted configurations are 15388677 and 3175152080 .

\section{Potential energy function, anharmonic vibrational and Franck-Condon factor calculations}


334 and 320 energies were computed at the CASSCF/MRCI-F12/CVQZ-F12 level (the As $3 \mathrm{~d}^{10}$ electrons being active in the MRCI-F12 calculations as described above) for the $\widetilde{\mathrm{X}}^{1} \mathrm{~A}^{\prime}$ and $\widetilde{\mathrm{A}}^{1} \mathrm{~A}^{\prime \prime}$

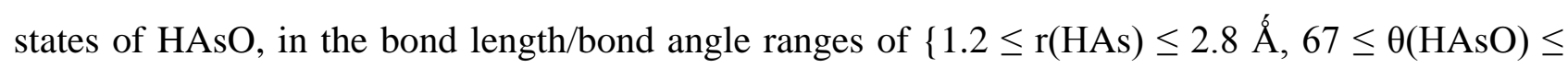

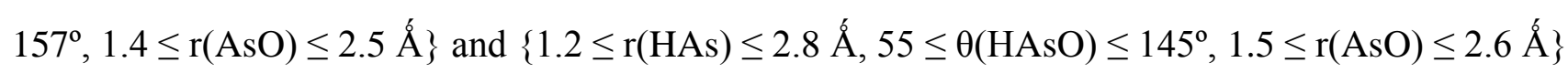
respectively. These energies were fitted to potential energy functions (PEFs) of a polynomial form. $^{7,13}$ Anharmonic vibrational wavefunctions (expressed as linear combinations of harmonic oscillator functions) and their corresponding energies were computed employing these PEFs. ${ }^{7,8}$ FCFs including anharmonicity and Duschinsky rotation were then calculated as described previously (see references 7,8 and 13 for details). Vibrational components in the $\widetilde{\mathrm{A}}^{1} \mathrm{~A}^{\prime \prime}-\widetilde{\mathrm{X}}^{1} \mathrm{~A}^{\prime} \mathrm{SVL}$ emission spectra of $\mathrm{HAsO} / \mathrm{DAsO}$ were simulated employing computed anharmonic FCFs and a transition frequency raised to the power 4, with Gaussian line shapes and a full-width-at-halfmaximum of $10 \mathrm{~cm}^{-1}$. In order to improve the agreement between the simulated and experimental SVL emission spectra, the iterative Franck-Condon analysis (IFCA) procedure, ${ }^{7,13}$ where some geometrical parameters used in the spectral simulation were varied, was also carried out (infra vide).

\section{Results and discussion}

\section{Optimized geometrical parameters}

The optimized geometrical parameters of the $\widetilde{\mathrm{X}}^{1} \mathrm{~A}^{\prime}$ and $\widetilde{\mathrm{A}}^{1} \mathrm{~A}^{\prime \prime}$ states of $\mathrm{HAsO}$ and computed excitation energies $\left(\Delta \mathrm{E}_{\mathrm{e}}\right.$ or $\left.\mathrm{T}_{0}\right)$ obtained at different levels of calculations are summarized in Table 2. Generally, the bond angles of the $\widetilde{\mathrm{X}}^{1} \mathrm{~A}^{\prime}$ and $\widetilde{\mathrm{A}}^{1} \mathrm{~A}^{\prime \prime}$ states of $\mathrm{HAsO}$ computed at different levels of MR theories obtained in the present study as shown in Table 2 are very consistent, while the computed bond lengths have a slightly wider range of values. For the $\widetilde{\mathrm{X}}^{1} \mathrm{~A}^{\prime}$ state of HAsO, which is a closed-shell electronic state, in addition to MR calculations, $\operatorname{CCSD}\left(\mathrm{T}^{*}\right)-\mathrm{F} 12$ calculations were also performed. It can been seen from the optimized geometrical parameters of the $\widetilde{\mathrm{X}}^{1} \mathrm{~A}^{\prime}$ state shown in Table 2 (with the VQZ-F12 basis sets) that, among the three MR methods employed, those obtained from the MRCI-F12 calculations generally agree best with those from the 
CCSD(T*)-F12 calculations, suggesting that the MRCI-F12 results are superior to the NEVPT2 and RSPT2-F12 results. Actually, it is pleasing to see that, the MRCI-F12/VQZ-F12 geometrical parameters are very close to the CCSD(T*)-F12/VQZ-F12 ones for the $\widetilde{\mathrm{X}}^{1} \mathrm{~A}^{\prime}$ state. With the CVQZF12 basis set and As $3 \mathrm{~d}^{10}$ being correlated, the optimized AsO bond lengths and HAsO bond angles obtained by the MRCI-F12 and $\operatorname{CCSD}\left(\mathrm{T}^{*}\right)-\mathrm{F} 12$ methods are also very close to each other (within $0.001 \AA$ and $0.13^{\circ}$, respectively), though their computed HAs bond lengths differ by $0.0096 \AA$. Comparing the computed bond angles of the $\widetilde{\mathrm{X}}^{1} \mathrm{~A}^{\prime}$ state obtained here with the previously computed B3LYP and $\operatorname{CCSD}(\mathrm{T})$ values, and the experimentally derived $r_{0}\{$ the $(0,0,0)$ vibrational level $\}$ value from reference 1 , the agreement is very good (within 1 degree). However, for the bond angle of the $\widetilde{\mathrm{A}}^{1} \mathrm{~A}^{\prime \prime}$ state, the agreement is less good, with the theoretical values from reference 1 slightly larger than our values. Specifically, our MR values are between 89.2 and $90.1^{\circ}$, while the B3LYP and $\operatorname{CCSD}(\mathrm{T})$ values from reference 1 are 91.7 and $91.5^{\circ}$ respectively, and their experimentally derived $\mathrm{r}_{0}$ value is $93.1 \pm 1.0^{\circ}$. Since the optimized geometrical parameters from calculations are $\mathrm{r}_{\mathrm{e}}$ values, while the experimentally derived counterparts are $r_{0}$ values, the comparison between theory and experiment of the geometrical parameters of the two states of $\mathrm{HAsO}$ will be further considered later, after the simulated and experimental SVL emission spectra are compared in the IFCA procedure. At this point, it should be noted that our MR methods should be more appropriate for the open-shell singlet $\widetilde{\mathrm{A}}^{1} \mathrm{~A}^{\prime \prime}$ state than the single-reference (SR) B3LYP and CCSD(T) methods, which presumably have employed unrestricted-spin wavefunctions for this open-shell singlet state, which will be affected by spin-contamination.

\section{Computed excitation energies}

Comparing the computed relative electronic energy $\left(\Delta \mathrm{E}_{\mathrm{e}}\right)$ of the $\widetilde{\mathrm{X}}^{1} \mathrm{~A}^{\prime}$ and $\widetilde{\mathrm{A}}^{1} \mathrm{~A}^{\prime \prime}$ states of HAsO obtained in the present study with the experimental $\mathrm{T}_{0}$ value of $1.899 \mathrm{eV}\left(15316.7 \mathrm{~cm}^{-1}\right)$ measured in the LIF spectrum of reference 1, the NEVPT2 and RSPT2-F12 values of $1.675 \mathrm{eV}$ $\left(13511.9 \mathrm{~cm}^{-1}\right)$ and $1.577 \mathrm{eV}\left(12719.9 \mathrm{~cm}^{-1}\right)$ obtained in the present study are too small (see Table 
2), as are the B3LYP and CCSD(T) values of $1.45 \mathrm{eV}\left(11731 \mathrm{~cm}^{-1}\right)$ and $1.764 \mathrm{eV}\left(14225 \mathrm{~cm}^{-1}\right)$ from reference 1. Nevertheless, the computed MRCI-F12 $\Delta \mathrm{E}_{\mathrm{e}}$ values of $1.934 \mathrm{eV}\left(15597.1 \mathrm{~cm}^{-1}\right)$ and $1.974 \mathrm{eV}\left(15918.8 \mathrm{~cm}^{-1}\right)$ obtained with the VQZ-F12 and CVQZ-F12 (correlating also As $3 \mathrm{~d}^{10}$ ) basis sets, respectively, agree much better with the experimental $\mathrm{T}_{0}$ value. Specifically, including zero-point energy corrections ( $\triangle \mathrm{ZPE}$; see footnote e of Table 2), the corresponding MRCI-F12 $\mathrm{T}_{0}$ values of $1.894 \mathrm{eV}\left(15273.4 \mathrm{~cm}^{-1}\right)$ and $1.934 \mathrm{eV}\left(15595.1 \mathrm{~cm}^{-1}\right)$ differ from the experimental $\mathrm{T}_{0}$ value by $-0.005 \mathrm{eV}\left(-43.3 \mathrm{~cm}^{-1}\right)$ and $+0.035 \mathrm{eV}\left(+278.4 \mathrm{~cm}^{-1}\right)$, respectively. Similarly for DAsO, the MRCI-F12 $\mathrm{T}_{0}$ values of $1.900 \mathrm{eV}\left(15326.8 \mathrm{~cm}^{-1}\right)$ and $1.940 \mathrm{eV}\left(15648.5 \mathrm{~cm}^{-1}\right)$ differ from the corresponding experimental value of $1.903 \mathrm{eV}\left(15348.9 \mathrm{~cm}^{-1}\right)$ of reference 1 by $+0.003 \mathrm{eV}(22.1$ $\left.\mathrm{cm}^{-1}\right)$ and $-0.037 \mathrm{eV}\left(-299.6 \mathrm{~cm}^{-1}\right)$. Summarizing, the differences between MRCI-F12 theory and experiment on the $\mathrm{T}_{0}$ values of the $\widetilde{\mathrm{X}}^{1} \mathrm{~A}^{\prime}-\widetilde{\mathrm{A}}^{1} \mathrm{~A}^{\prime \prime}$ band systems of $\mathrm{HAsO}$ and DAsO are now within the chemical accuracy of $1 \mathrm{kcal} . \mathrm{mol}^{-1}\left(0.043 \mathrm{eV} ; 349.8 \mathrm{~cm}^{-1}\right)$. The MRCI-F12 level of theory is able to give reliable relative electronic energy for the $\widetilde{\mathrm{X}}^{1} \mathrm{~A}^{\prime}-\widetilde{\mathrm{A}}^{1} \mathrm{~A}^{\prime \prime}$ band systems of $\mathrm{HAsO}$ and DAsO, while other levels of theory, including the MR NEVPT2 and RSPT2-F12 methods, are inadequate. In conclusion, we have achieved the objective of computing the relative electronic energy of the $\widetilde{\mathrm{X}}^{1} \mathrm{~A}^{\prime}$ - $\widetilde{\mathrm{A}}^{1} \mathrm{~A}^{\prime \prime}$ band system of HAsO accurately by employing the MRCI-F12 method with quadruplezeta quality basis sets.

\section{Computed vibrational frequencies}

The computed harmonic and fundamental vibrational frequencies of the $\widetilde{\mathrm{X}}^{1} \mathrm{~A}^{\prime}$ and $\widetilde{\mathrm{A}}^{1} \mathrm{~A}^{\prime \prime}$ states of HAsO and DAsO obtained in the present study are summarized in Table 3 together with available computed and experimental values. Before comparing them, the following points should be noted. First, previously computed vibrational frequencies are all harmonic values. ${ }^{1,15}$ Second, experimentally measured values are fundamental values. Nevertheless, in reference 1 , the harmonic values $\left(\omega^{0}\right)$ of the AsO stretching and $\mathrm{HAsO} / \mathrm{DAsO}$ bending modes of both states of $\mathrm{HAsO} / \mathrm{DAsO}$ were derived by fitting measured vibrational separations in these two progressions to an anharmonic 
formula. ${ }^{36}$ Third, the only vibrational frequency available experimentally for the HAs stretching mode of the $\widetilde{\mathrm{X}}^{1} \mathrm{~A}^{\prime}$ state of HAsO is from an IR matrix study and is a tentative assignment. ${ }^{16}$ There is no experimental vibrational frequency available for the HAs and DAs stretching modes of the $\widetilde{\mathrm{A}}^{1} \mathrm{~A}^{\prime \prime}$ state of HAsO and DAsO. Last, we have computed both harmonic and fundamental values for all three vibrational modes of the two states of $\mathrm{HAsO}$ and $\mathrm{DAsO}$, and also computed their $\mathrm{r}_{0}$ geometrical parameters (infra vide).

Since "raw" experimental vibrational frequencies are always fundamental values, it is appropriate to compare them with our computed fundamental values. Generally, all computed fundamental values are slightly larger than the available corresponding experimental values, except for the AsO stretching mode in the $\widetilde{\mathrm{A}}^{1} \mathrm{~A}^{\prime \prime}$ state of $\mathrm{DAsO}$, where the computed value is $1.2 \mathrm{~cm}^{-1}$ smaller than the experimental value. The generally slightly larger computed values may suggest slight underestimations of anharmonicities in our anharmonic vibrational frequency calculations. Specifically, for the AsO stretching modes of both states of HAsO and DAsO, our computed fundamental values agree reasonably well (within $\sim 16 \mathrm{~cm}^{-1}$ for both states) with the measured values, especially for DAsO (within $9 \mathrm{~cm}^{-1}$ for both states). For the HAsO bending modes, the agreement is not as good, but is within $27 \mathrm{~cm}^{-1}$, which may be considered as acceptable. For the HAs and DAs stretching modes of both states of HAsO and DAsO, they are not observed in both the LIF and SVL emission spectra of reference 1. As mentioned above, the only available experimental value is $1931 \mathrm{~cm}^{-1}$ for the $\widetilde{\mathrm{X}}^{1} \mathrm{~A}^{\prime}$ state of HAsO from a tentative assignment in an IR matrix study. ${ }^{16}$ Our computed fundamental value of $2030 \mathrm{~cm}^{-1}$ is $\sim 100 \mathrm{~cm}^{-1}$ larger than the available experimental value. In view of possible matrix effects on the experimental value and the likely underestimation of anharmonicity in the computed fundamental value of the HAs stretching mode (expected to be strongly anharmonic), it may be concluded that the agreement is reasonably acceptable and supports the experimental assignment in the matrix IR study. In addition, it is noted that if the MRCI-F12 anharmonic effect on the HAs stretching mode of $-80 \mathrm{~cm}^{-1}$ is used with the 
$\operatorname{CCSD}(\mathrm{T})$ harmonic value of $2027 \mathrm{~cm}^{-1}$ from reference 1 (see Table 3), a fundamental value of 1947 $\mathrm{cm}^{-1}$ is obtained, which agrees quite well with the IR experimental value ${ }^{16}$ of $1931 \mathrm{~cm}^{-1}$.

\section{Simulated spectra}

Some representative simulated $\widetilde{\mathrm{A}}^{1} \mathrm{~A}^{\prime \prime}(0,0,0) \rightarrow \widetilde{\mathrm{X}}^{1} \mathrm{~A}^{\prime}$ SVL emission spectra of HAsO and DAsO are shown in Figures 1 and 2 respectively. The corresponding experimental spectra from reference 1 are also given in these figures (bottom traces) for comparison. First, when the simulated spectra (middle row on the right in Figures 1 and 2) obtained using the computed $r_{e}$ geometrical parameters (from the MRCI-F12/CVQZ-F12 PEFs, see Table 2; i.e. the "pure" theoretical spectra with no empirical adjustment on any of the geometrical parameters used in the simulation) are compared with the corresponding experimental spectra, the overall agreement in the general vibrational structure is reasonably good. A slight difference is observed in the lower intensity of the experimental spectra towards larger displacement energy (smaller emission energy), when compared with the simulated ones. In any case, it can be concluded that the good general agreement between theory and experiment confirms the assignments of the carrier, the electronic states involved and the observed vibrational structures given in reference 1.

We attempted to improve the agreement between the simulated and experimental spectra via the IFCA procedure. This can be summarized as follows: If the experimental $r_{e}$ geometry of one electronic state (usually the ground state) is available, it is fixed, while the geometrical parameters of the other electronic state is varied systematically, until the best match between the simulated and experimental spectra is achieved. However, in the present case, no experimental $r_{e}$ geometrical parameters of either state of interest is available. Instead, only the $r_{0}$ geometrical parameters of the two electronic states of $\mathrm{HAsO}$ are available, which were derived from rotational analysis of the LIF $0_{0}{ }^{0}$ band in reference 1 (see Table 2). Since $r_{e}$ geometrical parameters are used in the IFCA procedure and we have computed $r_{e}$ and $r_{0}$ geometrical parameters from our PEFs for both the $\widetilde{\mathrm{X}}^{1} \mathrm{~A}^{\prime}$ and $\widetilde{\mathrm{A}}^{1} \mathrm{~A}^{\prime \prime}$ states, sets of experimentally derived $\mathrm{r}_{\mathrm{e}}$ geometrical parameters can be estimated by 
combining the experimentally derived $\mathrm{r}_{0}$ parameters from reference 1 with the differences between the corresponding computed $r_{e}$ and $r_{0}$ geometrical parameters from the present work. The MRCIF12/CVQZ-F12 PEFs for both the $\widetilde{\mathrm{X}}^{1} \mathrm{~A}^{\prime}$ and $\widetilde{\mathrm{A}}^{1} \mathrm{~A}^{\prime \prime}$ states were used for this procedure, as an $\mathrm{MR}$ method is needed for the states involved and the MRCI-F12 method gives the best agreement with the experimental $T_{0}$ value. These experimentally derived $r_{e}$ values are denoted as "derived $r_{e}$ (combining theory and experiment)" in Table 2 (or simply "combined $\mathrm{r}_{\mathrm{e}}$ "). Using the sets of combined $r_{e}$ geometrical parameters for both states, the simulated spectra are shown in the top traces on the left in Figures 1 and 2 for $\mathrm{HAsO}$ and DAsO respectively. Comparing them with the corresponding experimental spectra, the relative intensities of the vibrational structures in the simulated spectra decrease towards larger displacement energy as observed experimentally. They give better agreements between theory and experiments in the intensities of the overall structures than the "pure" theoretical spectra discussed above. However, the agreement in the relative intensities of the vibrational components within the various multiplet structures of the band seems to be slightly poorer.

Further IFCA calculations were carried out by fixing the combined $r_{e}$ geometrical parameters of the $\widetilde{\mathrm{X}}^{1} \mathrm{~A}^{\prime}$ state, while varying the combined $\mathrm{r}_{\mathrm{e}}$ geometrical parameters of the $\widetilde{\mathrm{A}}^{1} \mathrm{~A}^{\prime \prime}$ state systematically, until a best match between the simulated and experimental spectra was obtained. Since no HAs stretching mode was observed in the SVL emission spectra of HAsO and $\mathrm{DAsO}$, the main geometrical parameters to be varied were the $\mathrm{AsO}$ bond length, $\mathrm{r}_{\mathrm{e}}(\mathrm{AsO})$, and the HAsO bond angle, $\theta_{\mathrm{e}}(\mathrm{HAsO})$, of the upper state. However, it was found that varying these two geometrical parameters individually could lead to very similar effects on the simulated spectra. These are shown in the two simulated spectra in Figures 1 and 2 (top right and middle left traces) obtained using two sets of adjusted geometrical parameters for the $\widetilde{\mathrm{A}}^{1} \mathrm{~A}^{\prime \prime}$ state of HAsO/DAsO. It can be seen that they are quite similar. They also give the best overall match with the corresponding experimental spectra in terms of both the relative intensities of the vibrational components within sets of multiplets and the overall relative intensity changes over the whole bands. Specifically, 
further adjustments lead to slightly worse agreement in either the multiplet structures or the overall intensities over the band. Also, the simulated spectra using $\mathrm{r}_{\mathrm{e}}(\mathrm{AsO})=1.735 \AA$ and $\theta_{\mathrm{e}}(\mathrm{HAsO})=$ $91.5^{\circ}$ for the $\widetilde{\mathrm{A}}^{1} \mathrm{~A}^{\prime \prime}$ state (middle row left traces in Figures 1 and 2) may be considered as giving a very marginally better match with the experimental spectra than the simulated spectra using $\mathrm{r}_{\mathrm{e}}(\mathrm{AsO})$ $=1.74 \AA$ and $\theta_{\mathrm{e}}(\mathrm{HAsO})=91.0^{\circ}$ (top row right traces in Figures 1 and 2 ). For the sake of simplicity, the former simulated spectra are considered as the best simulated spectra from here onward. In any case, the vibrational structures at higher displacement energy regions $\left(>1500 \mathrm{~cm}^{-1}\right.$, corresponding to $<13816.7 \mathrm{~cm}^{-1}$ emission energy or $>723.8 \mathrm{~nm}$ emission wavelength) of the best simulated spectra are still slightly stronger than those in the experimental spectra. However, as mentioned, any further changes in the geometrical parameters of the upper state only make the match with experiment worse.

It should be noted that in reference 1, the dispersed fluorescence was detected by a cooled, red-sensitive photomultiplier (RCA C31034A). From available responsivity characteristic curves of this type of GaAs photocathodes, the responsivity begins to decrease towards low emission energy at the region of $\sim 800 \mathrm{~nm}\left(\sim 12500 \mathrm{~cm}^{-1}\right)$. Specifically, a rapid cut off starts from $\sim 850 \mathrm{~nm}(\sim 11764$ $\left.\mathrm{cm}^{-1}\right) .{ }^{37}$ With an experimental $\mathrm{T}_{0}$ value of $15316.7 \mathrm{~cm}^{-1}$ from the LIF spectrum, the region in the SVL emission spectrum, where intensity is reduced due to the variation in the detector responsivity, is expected to be at $\sim 2820 \mathrm{~cm}^{-1}$ displacement energy, while the rapid cut off has a displacement energy of $\sim 3550 \mathrm{~cm}^{-1}$. The fact that the SVL emission spectra reported in reference 1 have displacement energies up to $\sim 3500 \mathrm{~cm}^{-1}$ is in line with the rapid cut off of the detector responsivity at $\sim 3550 \mathrm{~cm}^{-1}$. In view of the above considerations, the high displacement energy (low emission energy) regions in the experimental SVL emission spectra of $\mathrm{HAsO}$ and DAsO are expected to be affected by the decrease in the responsivity in the cut off region of the detector used to record them. In this connection, it is concluded that the discrepancies between the experimental and best simulated spectra are almost certainly due to the detector used. 
Summarizing, from IFCA, there appears to be more than one unique set of geometrical parameters for the upper state which could give the best match between simulated and experimental spectra. Although the differences in $\mathrm{r}_{\mathrm{e}}(\mathrm{AsO})$ and $\theta_{\mathrm{e}}(\mathrm{HAsO})$ between these sets are small, the favoured IFCA parameters for the $\widetilde{\mathrm{A}}^{1} \mathrm{~A}^{\prime \prime}$ state are $\mathrm{r}_{\mathrm{e}}(\mathrm{AsO})=1.735 \AA$ and $\theta_{\mathrm{e}}(\mathrm{HAsO})=91.5^{\circ}$. Also, the high displacement energy (low emission energy) regions of the experimental SVL emission spectra of HAsO and DAsO reported in reference 1 are most likely affected by the loss of responsivity of the detector near the cut off region.

\section{$R_{\mathrm{e}} / \mathbf{r}_{0}$ geometrical parameters, their rotational constants and their changes upon excitation}

In view of the IFCA results, the $r_{e}$ and $r_{0}$ geometrical parameters of the two states of $\mathrm{HAsO} / \mathrm{DAsO}$ are considered again. Before they are discussed, it should be noted that some general considerations on the relationships between $r_{e}$ and $r_{0}$ geometrical parameters, their derivations and relationships with the corresponding rotational constants have been discussed in detail previously in a similar study on the SVL emission of HPO and DPO, ${ }^{13}$ and hence will not be repeated here. Readers should refer to reference 13 for some approximations and limitations involved in deriving them both experimentally and computationally. In addition, since the computed NEVPT2 and RSPT2-F12 relative electronic energies are rather poor, when compared with the experimental value as discussed, their geometrical parameters will be largely ignored in the comparisons of derived geometrical parameters given below. Regarding IFCA and comparisons between simulated and experimental spectra, since it is the changes in the $r_{e}$ geometrical parameters upon excitation, which determine the computed FCFs, the changes of the geometrical parameters between the $\widetilde{\mathrm{X}}^{1} \mathrm{~A}^{\prime}$ and $\widetilde{\mathrm{A}}^{1} \mathrm{~A}^{\prime \prime}$ states of HAsO obtained by different methods are compiled in Table 4 . At the same time, since $\mathrm{r}_{0}$ geometrical parameters from reference 1 were calculated from $\mathrm{r}_{0}$ rotational constants, which were derived from matching simulated and observed rotational structures (similar to the IFCA approach used here for the vibrational structure), rotational constants obtained in different ways are given in Table 5. It should be noted that, for this type of $C_{S}$ triatomic molecules, a set of 
geometrical parameters gives a unique set of rotational constants, but not vice versa (see reference 13). All the rotational constants shown in Table 5 were evaluated from the corresponding geometrical parameters, except the $\mathrm{r}_{0}$ rotational constants from reference 1 , which were derived from rotational analysis.

From Table 4, although IFCA does not involve the HAs bond length, as no HAs/DAs stretching mode was observed in the SVL emission spectra of HAsO/DAsO, when the combined $\Delta \mathrm{r}_{\mathrm{e}}(\mathrm{HAs})$ value $(-0.008 \AA)$ between the $\widetilde{\mathrm{X}}^{1} \mathrm{~A}^{\prime}$ and $\widetilde{\mathrm{A}}^{1} \mathrm{~A}^{\prime \prime}$ states and experimentally derived $\Delta \mathrm{r}_{0}(\mathrm{HAs})$ value (-0.004 $\AA$ ) from reference 1 are compared with the corresponding computed values obtained by different methods, the MRCI-F12/CVQZ-F12 $\Delta \mathrm{r}_{\mathrm{e}}(\mathrm{HAs})$ and $\Delta \mathrm{r}_{0}(\mathrm{HAs})$ values $\{-$ 0.0078(optimized)/-0.0063(PEFs) and -0.0032 $\mathrm{A}\}$ agree best. For the AsO bond length and HAsO bond angle, the IFCA $\Delta \mathrm{r}_{\mathrm{e}}(\mathrm{AsO})$ and $\Delta \theta_{\mathrm{e}}(\mathrm{HAsO})$ values of $0.1047 \AA$ and $-9.8^{\circ}$ are smaller than the corresponding combined $\Delta \mathrm{r}_{\mathrm{e}}$ and $\Delta \theta_{\mathrm{e}}$ values of $0.1204 \AA$ and $-8.6^{\circ}$, obtained based on the LIF experimentally derived $r_{0}$ values from reference 1 , by $0.0157 \AA$ and $1.2^{\circ}$ respectively. Comparing the combined $\Delta \mathrm{r}_{\mathrm{e}}(\mathrm{AsO})$ value $(0.1204 \AA)$ with the corresponding MRCI-F12 values with the two basis sets used $(0.1263$ and $0.1242 \AA)$, it can be seen that they agree quite well. However, the MRCI-F12 $\Delta \theta_{\mathrm{e}}$ values are larger than the combined $\Delta \theta_{\mathrm{e}}$ value by $\sim 3^{\circ}$ and the corresponding IFCA value by $\sim 2^{\circ}$. Summarizing, computed MRCI-F12/CVQZ-F12 $\Delta \mathrm{r}_{\mathrm{e}}$ values agree very well with the corresponding combined values obtained based on the rotational analyses of reference 1 , but the MRCI-F12/CVQZ-F12 $\Delta \theta_{\mathrm{e}}$ value is smaller than the combined value by $\sim 3^{\circ}$, though it is closer to the IFCA value (differing by $\sim 2^{\circ}$ ). Regarding the IFCA geometry changes, since the experimental SVL emission spectra from reference 1 have most likely been affected by a loss of responsivity with the detector near the cut off region as discussed above, it would not be meaningful to pursue in this direction further, until more reliable experimental SVL emission spectra are available. Regarding the combined $r_{e}$ geometrical parameters, they were estimated based on the $r_{0}$ geometrical parameters from reference 1 , which were calculated using $r_{0}$ rotational constants derived from 
rotational analyses of the LIF $0_{0}{ }^{0}$ band. In this connection, rotational constants obtained by different methods are given in Table 5 and discussed below.

From Table 5, for both the $\widetilde{\mathrm{X}}^{1} \mathrm{~A}^{\prime}$ and $\widetilde{\mathrm{A}}^{1} \mathrm{~A}^{\prime \prime}$ states, the values of both $\mathrm{r}_{\mathrm{e}}$ and $\mathrm{r}_{0}$ rotational constants $\mathrm{B}$ and $\mathrm{C}$ are reasonably consistent, while those of rotational constant $\mathrm{A}$ have a wider spread. This is very similar to our previous findings for HPO. ${ }^{13}$ The uncertainties involved in converting rotational constants to geometrical parameters and vice versa have been discussed in reference 13 for this type of $\mathrm{C}_{\mathrm{S}}$ triatomic molecules. In addition to these limitations and uncertainties, in the present case, it has been found that similar computed FCFs could be obtained by different combinations of $\mathrm{r}_{\mathrm{e}}(\mathrm{AsO})$ and $\theta_{\mathrm{e}}(\mathrm{HAsO})$ values. Consequently, it is not possible to obtain unique sets of geometrical parameters reliably for the two states of $\mathrm{HAsO}$ with available experimental and computational data.

\section{Concluding remarks}

We have carried out various MR wavefunction calculations on the $\widetilde{\mathrm{X}}^{1} \mathrm{~A}^{\prime}$ and $\widetilde{\mathrm{A}}^{1} \mathrm{~A}^{\prime \prime}$ states of HAsO. It was found that both the NEVPT2 and RSPT2-F12 methods are inadequate for their relative electronic energies, showing the high demands in theory for these electronic systems. Nevertheless, it is pleasing that the MRCI-F12 method has yielded reliable relative electronic energies for these two states of $\mathrm{HAsO}$, thus achieving a major goal of the present investigation. However, if one pushes for high accuracies, it may be noticed that the computed frozen core (FC) MRCI-F12/VQZ-F12 $\mathrm{T}_{0}$ value of $1.894 \mathrm{eV}\left(15273.4 \mathrm{~cm}^{-1}\right)$ agrees slightly better than the MRCIF12/CVQZ-F12 value, including the As $3 \mathrm{~d}^{10}$ electrons, of $1.934 \mathrm{eV}\left(15595.1 \mathrm{~cm}^{-1}\right)$ with the LIF experimental value of $1.899 \mathrm{eV}\left(15316.7 \mathrm{~cm}^{-1}\right) .{ }^{1}$ This seems to be contrary to theory in that, including the As $3 \mathrm{~d}^{10}$ electrons should be at a higher level than frozen core. Of course, some cancellation of errors, such as, due to size-inconsistency of the MRCI method and/or the neglect of higher order excitations, may be the cause. In addition, it should be noted that the MRCIF12/CVQZ-F12 calculations, which have the As $3 \mathrm{~d}^{10}$ electrons active, have employed a value of 1.5 
for the geminal Slater exponent, instead of the default value of 1.0 for valence only calculations as employed in the FC calculations. The very slightly worse performance of the MRCI-F12/CVQZF12 calculations on relative electronic energies may be due to the use of 1.5 for the geminal Slater exponent, because strictly speaking, this value is optimized for a full core calculation, which should include also the As $3 s^{2} 3 p^{6}$ and $\mathrm{O} 1 \mathrm{~s}^{2}$ electrons. However, a full core MRCI-F12 calculation for HAsO will be beyond our computational capacity. In any case, generally for explicitly correlated calculations, in addition to the choice of basis sets, the geminal Slater exponent is an additional parameter to consider, though it is beyond the scope of the present investigation to obtain an optimized value for including only the As $3 \mathrm{~d}^{10}$ electrons. Nevertheless, the computed geometrical parameters obtained in the CVQZ-F12 calculations, which have correlated the As $3 \mathrm{~d}^{10}$ electrons explicitly, are clearly different from those of the FC calculations, and the former values appear to give better changes upon excitation, when compared to available experimentally derived values, than the FC ones, as discussed.

From the comparisons between simulated and experimental SVL emission spectra of $\mathrm{HAsO} / \mathrm{DAsO}$ in the IFCA procedure, it has been found that the set of $\mathrm{r}_{\mathrm{e}}$ geometrical parameters used, which give the best match, may not be unique. This is similar to what has been discussed previously - that converting rotational constants to geometrical parameters may not lead to a unique set of values. ${ }^{13}$ Although there are limitations in deriving reliable geometrical parameters by comparing simulated and experimental spectra as discussed above and previously, ${ }^{13}$ the good general agreement between simulated and experimental spectra reported in the present study clearly supports the assignments of the carrier, the electronic states and vibrational structure of the experimental SVL emission spectra reported in reference 1. In addition, our spectral simulations suggest that the experimental spectra suffer from a loss of responsivity near the cut off region of the detector used to record them. The multi-reference methods used in this work, MRCI-F12/VQZ-F12 
and MRCI-F12/CVQZ-F12, both give $\mathrm{T}_{0}$ values within chemical accuracy $\left(1 \mathrm{kcal}^{\mathrm{mol}}{ }^{-1} ; 0.043 \mathrm{eV}\right)$ of the experimental $T_{0}$ value for the $\widetilde{\mathrm{A}}^{1} \mathrm{~A}^{\prime \prime}-\widetilde{\mathrm{X}}^{1} \mathrm{~A}^{\prime}$ transition, which is a clear improvement over previous calculations.

Acknowledgement: The authors are grateful to the RGC of HKSAR (GRF Grants PolyU 5018/13P and PolyU 153013/15P) for support. Computations were carried out using resources of the NSCCS, EPSRC (UK). 
Table 1. Basis sets (relativistic effective core potential, ECP10MDF, for As) used in CASSCF/RSPT2-F12, CASSCF/MRCI-F12 and RHF/CCSD(T*)-F12 calculations with the default frozen core for $\mathrm{O}$ and As (only the $4 \mathrm{~s}^{2} 4 \mathrm{p}^{3}$ electrons of As were correlated; see text for basis sets used, when As $3 \mathrm{~d}^{10}$ electrons were also correlated).

\begin{tabular}{|l|l|l|l|}
\hline Basis & $\mathrm{As}^{\mathrm{a}}$ & O and H & Nbasis $^{\mathrm{b}}$ \\
\hline $\mathrm{AO}^{\mathrm{c}}$ & VQZ-PP-F12 & Default VQZ-F12 & 226 \\
\hline DF $^{\mathrm{d}}$ & VQZ-PP-F12_MP2FIT(spdfg) & Default AVQZ_MP2FIT & 492 \\
\hline JK $^{\mathrm{e}}$ & def2-AQZVPP-JKFIT(spdfg) & Default AVQZ_JKFIT & 476 \\
\hline RI $^{\mathrm{f}}$ & VQZ-PP-F12-OPT(spdfgh) & VQZ-F12_OPT(spdfgh and spdfg) & 364 \\
\hline
\end{tabular}

${ }^{\mathrm{a}}$ The VQZ-PP-F12, VQZ-PP-F12_MPFIT and VQZ-PP-F12_OPT basis sets for As are optimized for F12 calculations with the ECP10MDF ECP for As (see reference 26).

${ }^{b}$ The number of contracted Gaussian functions of the basis set for HAsO.

${ }^{\mathrm{c}}$ Atomic orbital basis sets (from reference 32).

${ }^{\mathrm{d}}$ Density fitting basis sets (from reference 26).

e The JK basis sets are used as the density fitting basis for Fock and exchange matrices (see MOLPRO online manual ${ }^{17}$ and references therein). The def2-AQZVPP-JKFIT basis set for As is from reference 27 .

${ }^{\mathrm{f}}$ Resolution of the identity basis sets; MOLPRO cannot find the RI (or OPTRI) basis sets for As, O and $\mathrm{H}$ from its basis set library. Consequently, the $\mathrm{H}$ (spdfg) and $\mathrm{O}$ (spdfgh) cc-pVQZ-F12_OPTRI basis sets (from reference 28) and the As cc-pVQZ-PP-F12-OPTRI (spdfgh) basis set (from reference 29) were taken from the EMSL Basis Set Exchange Library. ${ }^{30,31}$ 
Table 2. Computed geometrical parameters (bond lengths in $\AA$ and bond angle in degrees; $r_{e}$ values unless otherwise stated) and relative energies $\left\{\Delta \mathrm{E}_{\mathrm{e}}\right.$ in $\mathrm{eV}\left(\mathrm{cm}^{-1}\right)$; unless otherwise stated $\}$ of the $\widetilde{\mathrm{X}}^{1} \mathrm{~A}^{\prime}$ and $\widetilde{\mathrm{A}}^{1} \mathrm{~A}^{\prime \prime}$ states of $\mathrm{HAsO}$ (values for $\mathrm{DAsO}$ are labelled as such).

\begin{tabular}{|c|c|c|c|c|}
\hline$\widetilde{\mathrm{X}}^{1} \mathrm{~A}^{\prime}$ & $\mathrm{AsH}$ & $\mathrm{AsO}$ & HAsO & $\Delta \mathrm{E}_{\mathrm{e}}$ \\
\hline CCSD(T*)-F12b/VQZ-F12 ${ }^{\mathrm{a}}$ & 1.5629 & 1.6375 & 101.42 & \\
\hline NEVPT2/aug-cc-pVQZ, aug-cc-pVQZ-PP ${ }^{\mathrm{a}}$ & 1.5482 & 1.6479 & 101.27 & \\
\hline RSPT2-F12/VQZ-F12 & 1.5575 & 1.6410 & 101.57 & \\
\hline MRCI-F12/VQZ-F12 ${ }^{\mathrm{a}}$ & 1.5665 & 1.6382 & 101.75 & \\
\hline $\mathrm{CCSD}\left(\mathrm{T}^{*}\right)-\mathrm{F} 12 \mathrm{~b} / \mathrm{CVQZ}-\mathrm{F} 12^{\mathrm{b}}$ & 1.5521 & 1.6282 & 101.29 & \\
\hline MRCI-F12/CVQZ-F12b & 1.5425 & 1.6291 & 101.42 & \\
\hline From PEF $\mathrm{r}_{\mathrm{e}}(\mathrm{MRCI}-\mathrm{F} 12 / \mathrm{CVQZ}-\mathrm{F} 12)^{\mathrm{b}}$ & 1.5402 & 1.6290 & 101.57 & \\
\hline 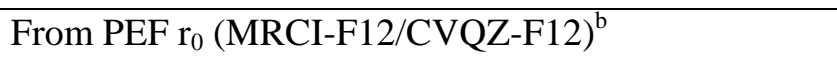 & 1.5567 & 1.6329 & 101.75 & \\
\hline DAsO: PEF r $_{0}\left(\right.$ MRCI-F12/CVQZ-F12) ${ }^{b}$ & 1.5521 & 1.6330 & 101.67 & \\
\hline B3LYP/aug-cc-pV5Z & 1.572 & 1.631 & 101.5 & \\
\hline CCSD(T)/aug-cc-pV5Zc & 1.544 & 1.631 & 101.1 & \\
\hline $\mathrm{HAsO}$, derived $\mathrm{r}_{0}$ from LIF $0_{0}{ }^{0}$ band $^{\mathrm{c}}$ & $1.573(3)$ & $1.6342(5)$ & $101.5(4)$ & \\
\hline $\mathrm{HAsO}$, derived $\mathrm{r}_{\mathrm{e}}$ (combine theory and experiment $)^{\mathrm{d}}$ & 1.557 & 1.630 & 101.3 & \\
\hline DAsO $r_{0}\left(\right.$ derived $r_{e}{ }^{d}$ plus DAsO computed $\left.r_{e} / r_{0}\right)$ & 1.569 & 1.630 & 101.4 & \\
\hline \multicolumn{5}{|l|}{$\widetilde{\mathrm{A}}^{1} \mathrm{~A}^{\prime \prime}$} \\
\hline NEVPT2/aug-cc-pVQZ, aug-cc-pVQZ-PP ${ }^{\mathrm{a}}$ & 1.5535 & 1.7691 & 89.19 & $1.675(13511.9)$ \\
\hline RSPT2-F12/VQZ-F12 $^{\text {a }}$ & 1.5556 & 1.7599 & 89.20 & $1.577(12719.9)$ \\
\hline MRCI-F12/VQZ-F12 ${ }^{\mathrm{a}}$ & 1.5472 & 1.7645 & 89.64 & $1.934(15597.1)$ \\
\hline$\Delta \mathrm{E}_{0}\left(\mathrm{~T}_{0}\right)^{\mathrm{a}, \mathrm{e}}$ & & & & $1.894(15273.4)$ \\
\hline$\Delta \mathrm{E}_{0}\left(\mathrm{~T}_{0}\right)^{\mathrm{a}, \mathrm{f}}(\mathrm{DAsO})$ & & & & $1.900(15326.8)$ \\
\hline MRCI-F12/CVQZ-F12b $r_{e}, T_{e}$ & 1.5347 & 1.7533 & 89.89 & $1.974(15918.8)$ \\
\hline From PEF (MRCI-F12/CVQZ-F12) ${ }^{b} r_{e}, T_{e}$ & 1.5339 & 1.7531 & 89.63 & $1.977(15942.6)$ \\
\hline From PEF (MRCI-F12/CVQZ-F12): ${ }^{\mathrm{b}} \mathrm{r}_{0}$ and $\Delta \mathrm{E}_{0}\left(\mathrm{~T}_{0}\right)^{\mathrm{e}}$ & 1.5535 & 1.7533 & 90.07 & $1.934(15595.1)$ \\
\hline
\end{tabular}




\begin{tabular}{|c|c|c|c|c|}
\hline DAsO: $\mathrm{PEF} \mathrm{r}_{0}(\mathrm{MRCI}-\mathrm{F} 12 / \mathrm{CVQZ}-\mathrm{F} 12)^{\mathrm{b}}$ and $\Delta \mathrm{E}_{0}\left(\mathrm{~T}_{0}\right)^{\mathrm{f}}$ & 1.5481 & 1.7549 & 89.95 & $1.940(15648.5)$ \\
\hline B3LYP/aug-cc-pV5Z ${ }^{c}$ & 1.545 & 1.745 & 91.7 & $1.45(11731)$ \\
\hline CCSD(T)/aug-cc-pV5Z & 1.522 & 1.733 & 91.5 & $1.764(14225)$ \\
\hline HAsO LIF $0_{0}{ }^{0}$ band: $^{\mathrm{c}} \mathrm{r}_{0}$ and $\mathrm{T}_{0}$ & $1.569(4)$ & $1.7509(9)$ & $93.1(10)$ & $1.899(15316.7)$ \\
\hline $\mathrm{HAsO}$, derived $\mathrm{r}_{\mathrm{e}}$ (combining theory and experiment) ${ }^{\mathrm{d}}$ & 1.549 & 1.7507 & 92.7 & \\
\hline DAsO $r_{0}\left(\right.$ derived $r_{e}{ }^{d}$ plus DAsO computed $\left.r_{e} / r_{0}\right)$ & 1.563 & 1.7525 & 93.0 & \\
\hline DAsO: LIF T ${ }_{0}^{c}$ & & & & $1.903(15348.9)$ \\
\hline IFCA $^{g} r_{e}$ & 1.549 & 1.735 & 91.5 & \\
\hline $\mathrm{IFCA}^{\mathrm{g}} \mathrm{r}_{0}^{\mathrm{h}}$ & 1.569 & 1.735 & 91.94 & \\
\hline DAsO: IFCA $^{g} r_{0}{ }^{i}$ & 1.563 & 1.737 & 91.82 & \\
\hline
\end{tabular}

${ }^{\mathrm{a}}$ At optimized geometries; see Table 1 and text for the details of the basis sets used; default frozen core for As and $\mathrm{O}$.

${ }^{\mathrm{b}}$ At optimized geometries; including As $3 \mathrm{~d}^{10}$ explicitly in the MRCI-F12 and CCSD(T*)-F12 calculations (frozen As $3 \mathrm{~s}^{2} 3 \mathrm{p}^{6}$ and $\mathrm{O} 1 \mathrm{~s}^{2}$ ); in addition to the basis sets described in Table 1, uncontracted 4d (exponents: 12.0, 4.0, 1.3333333, 0.444444), 2f (14.0, 6.0) and 2g $(9.6,2.4)$ functions were added in $\mathrm{AO}$ basis set of As to account for As $3 \mathrm{~d}^{10}$, giving totally 278 contracted basis functions in the AO basis set of HAsO (see text).

${ }^{\mathrm{c}}$ From reference 1.

${ }^{\mathrm{d}}$ Using the experimental $r_{0}$ values from reference 1 and the differences between computed $r_{0}$ and $r_{e}$ values (from MRCI-F12/CVQZ-F12 PEF) obtained from variational calculations of the anharmonic vibrational wavefunctions; see text.

${ }^{\mathrm{e}} \Delta \mathrm{E}_{0}=\Delta \mathrm{E}_{\mathrm{e}}+\Delta \mathrm{ZPE}$ (zero-point energy corrections $=-323.9 \mathrm{~cm}^{-1}=-0.040 \mathrm{eV}$, using computed fundamental frequencies from PEFs; see text).

${ }^{\mathrm{f}}$ As footnote e, but for DAsO $\left(\triangle \mathrm{ZPE}=-270.3 .9 \mathrm{~cm}^{-1}=-0.034 \mathrm{eV}\right.$, using computed fundamental frequencies from PEFs; see text). 
${ }^{g}$ The $r_{e}$ geometry of the $\widetilde{\mathrm{X}}^{1} \mathrm{~A}^{\prime}$ state and the $\mathrm{r}_{\mathrm{e}}(\mathrm{HAs})$ bond length of the $\widetilde{\mathrm{A}}^{1} \mathrm{~A}^{\prime \prime}$ state of HAsO were fixed to the corresponding combined $r_{e}$ values (see footnote $\left.d\right)$, while the $r_{e}(\mathrm{AsO})$ and $\theta_{\mathrm{e}}(\mathrm{HAsO})$ of the $\widetilde{\mathrm{A}}^{1} \mathrm{~A}^{\prime \prime}$ state were varied in the IFCA procedure (see text).

${ }^{\mathrm{h}}$ The IFCA $\mathrm{r}_{0}$ geometry of the $\widetilde{\mathrm{A}}^{1} \mathrm{~A}^{\prime \prime}$ state of $\mathrm{HAsO}$ was obtained using the IFCA $\mathrm{r}_{\mathrm{e}}$ geometry plus the differences between the computed $r_{0}$ and $r_{e}$ values obtained from the PEF of the $\widetilde{A}^{1} A^{\prime \prime}$ state (see footnote d) obtained from variational calculations of the anharmonic vibrational wavefunctions.

${ }^{\mathrm{i}}$ The IFCA $\mathrm{r}_{0}$ geometry of the $\widetilde{\mathrm{A}}^{1} \mathrm{~A}^{\prime \prime}$ state of DAsO was obtained using the IFCA $\mathrm{r}_{\mathrm{e}}$ geometry of HAsO plus the differences between the computed $\mathrm{r}_{0}$ DAsO and $\mathrm{r}_{\mathrm{e}} \mathrm{HAsO}$ values obtained from the PEF the $\widetilde{\mathrm{A}}^{1} \mathrm{~A}^{\prime \prime}$ state (see footnotes $\mathrm{d}$ and $\mathrm{h}$ ). 
Table 3. Computed harmonic [fundamental] vibrational frequencies (in $\mathrm{cm}^{-1}$ ) of the $\widetilde{\mathrm{X}}^{1} \mathrm{~A}^{\prime}$ and $\widetilde{\mathrm{A}}^{1} \mathrm{~A}^{\prime \prime}$ states of $\mathrm{HAsO}$ and DAsO.

\begin{tabular}{|c|c|c|c|c|c|c|}
\hline \multirow[t]{2}{*}{$\widetilde{\mathrm{X}}^{1} \mathrm{~A}^{\prime}$} & \multicolumn{3}{|c|}{$\mathrm{HAsO}$} & \multicolumn{3}{|c|}{ DAsO } \\
\hline & HAs & Bending & $\mathrm{AsO}$ & DAs & bending & $\mathrm{AsO}$ \\
\hline MRCI-F12 $^{\mathrm{a}}$ & $2110[2030]$ & $871[855]$ & $959[946]$ & 1504[1463] & $648[639]$ & $946[934]$ \\
\hline $\mathrm{B} 3 \mathrm{LYP}^{\mathrm{b}}$ & 1970 & 849 & 974 & 1405 & 627 & 965 \\
\hline $\operatorname{CCSD}(T)^{b}$ & 2027 & 864 & 961 & 1445 & 641 & 950 \\
\hline LIF (hot bands) & & {$[\sim 832.3]^{\mathrm{d}}$} & & & {$[\sim 627.4]^{\mathrm{d}}$} & {$[\sim 922]^{d}$} \\
\hline$S V L^{c, e}$ & & $837.0[832.4]$ & $944.4[937]$ & & $631.8[627.1]$ & $929.5[924.1]$ \\
\hline IR (Ar matrix) $)^{f}$ & [1931] & & & & & \\
\hline HF/ECP-DZP & 1963 & 859 & 986 & 1399 & 634 & 982 \\
\hline$\widetilde{\mathrm{A}}^{1} \mathrm{~A}^{\prime \prime}$ & & & & & & \\
\hline MRCI-F12 $^{\mathrm{a}}$ & 2030[1907] & $629[617]$ & $681[661]$ & 1446[1387] & $489[475]$ & $640[634]$ \\
\hline $\mathrm{B} 3 \mathrm{LYP}^{\mathrm{b}}$ & 2042 & 562 & 684 & 1454 & 421 & 667 \\
\hline $\operatorname{CCSD}(\mathrm{T})^{\mathrm{b}}$ & 2097 & 575 & 702 & 1493 & 433 & 682 \\
\hline $\mathrm{LIF}^{\mathrm{c}, \mathrm{e}}$ & & $604.6[599.4]$ & $648.9[644.9]$ & & $446.0[448.3]$ & $638.6[635.2]$ \\
\hline
\end{tabular}

${ }^{\mathrm{a}}$ From MRCI-F12/CVQZ-F12 PEF including As 3d ${ }^{10}$; present work.

${ }^{\mathrm{b}}$ From reference 1, using the aug-cc-pV5Z basis set.

${ }^{\mathrm{c}}$ From reference 1.

${ }^{\mathrm{d}}$ Averages of the few values given in reference 1 .

e The harmonic values $\left(\omega^{0}\right)$ were obtained by fitting to an anharmonic formula (reference 21 in reference 1$)^{36}$

${ }^{\mathrm{f}}$ Tentatively assigned to H-As stretching of HAsO; see reference 16.

${ }^{\mathrm{g}} \mathrm{HF} / \mathrm{ECP}-\mathrm{DZP}, 6-31 \mathrm{G}^{*}$ harmonic frequencies scaled uniformly by a factor of 0.9 ; see reference 15 . 
Table 4. Geometry changes (bond lengths in $\AA$ and bond angle in degrees) upon excitation from the $\widetilde{\mathrm{X}}^{1} \mathrm{~A}^{\prime}$ state to the $\widetilde{\mathrm{A}}^{1} \mathrm{~A}^{\prime \prime}$ state of HAsO (values for DAsO are labelled as such).

\begin{tabular}{|c|c|c|c|}
\hline Methods $^{\mathrm{a}}$ & $\Delta \mathrm{r}(\mathrm{AsH})$ & $\Delta \mathrm{r}(\mathrm{AsO})$ & $\Delta \theta(\mathrm{HAsO})$ \\
\hline NEVPT2/aug-cc-pVQZ, aug-cc-pVQZ-PP & +0.0053 & +0.1212 & -12.08 \\
\hline RSPT2-F12/VQZ-F12 & -0.0019 & +0.1189 & -12.37 \\
\hline MRCI-F12/VQZ-F12 & -0.0193 & +0.1263 & -12.11 \\
\hline MRCI-F12/CVQZ-F12 & -0.0078 & +0.1242 & -11.53 \\
\hline $\mathrm{PEF}_{\mathrm{e}}(\mathrm{MRCI}-\mathrm{F} 12 / \mathrm{CVQZ}-\mathrm{F} 12)$ & -0.0063 & +0.1241 & -11.94 \\
\hline $\mathrm{PEF} \mathrm{r}_{0}(\mathrm{MRCI}-\mathrm{F} 12 / \mathrm{CVQZ}-\mathrm{F} 12)$ & -0.0032 & +0.1204 & -11.68 \\
\hline DAsO $\mathrm{r}_{0}(\mathrm{MRCI}-\mathrm{F} 12 / \mathrm{CVQZ}-\mathrm{F} 12)$ & -0.0040 & +0.1219 & -11.72 \\
\hline B3LYP/aug-cc-pV5Z b & -0.027 & +0.114 & -9.8 \\
\hline CCSD(T)/aug-cc-pV5Z ${ }^{b}$ & -0.022 & +0.102 & -9.6 \\
\hline Experimental $r_{0}^{b}$ & -0.004 & +0.1167 & -8.4 \\
\hline Derived $r_{e}(\text { combine theory and experiment })^{c}$ & -0.008 & +0.1204 & -8.6 \\
\hline DAsO $\mathrm{r}_{0}\left(\right.$ derived $\mathrm{r}_{\mathrm{e}}$ plus DAsO computed $\left.\mathrm{r}_{\mathrm{e}} / \mathrm{r}_{0}\right)$ & -0.006 & +0.1221 & -8.4 \\
\hline IFCA $r_{e}{ }^{d}$ & -0.008 & +0.1047 & -9.8 \\
\hline IFCA $r_{0}{ }^{\mathrm{e}}$ & -0.004 & +0.1008 & -9.6 \\
\hline DAsO: IFCA $r_{0}$ & -0.006 & +0.1066 & -9.6 \\
\hline
\end{tabular}

${ }^{\mathrm{a}}$ See Table 2 and footnotes there.

${ }^{\mathrm{b}}$ From reference 1 .

${ }^{\mathrm{c}}$ See footnote $\mathrm{d}$ of Table 2

${ }^{d}$ The $r_{e}$ geometry of the $\widetilde{X}^{1} A^{\prime}$ state and the $r_{e}(H A s)$ bond length of the $\widetilde{A}^{1} A^{\prime \prime}$ state of HAsO were fixed to the corresponding combined $r_{e}$ values (see footnote $d$ of Table 2), while the $r_{e}(A s O)$ and $\theta_{\mathrm{e}}(\mathrm{HAsO})$ of the $\widetilde{\mathrm{A}}^{1} \mathrm{~A}^{\prime \prime}$ were varied (see footnote $\mathrm{g}$ of Table 2 and text).

e The $\mathrm{r}_{0}$ geometries of the $\widetilde{\mathrm{X}}^{1} \mathrm{~A}^{\prime}$ state from reference 1 and the IFCA $\mathrm{r}_{\mathrm{e}}$ geometry of the $\widetilde{\mathrm{A}}^{1} \mathrm{~A}^{\prime \prime}$ state plus the computed differences between $r_{e}$ and $r_{0}$ geometrical parameters from PEFs. 
Table 5. Rotational constants ( $\mathrm{r}_{\mathrm{e}}$ values in $\mathrm{cm}^{-1}$; unless otherwise stated) of the $\widetilde{\mathrm{X}}^{1} \mathrm{~A}^{\prime}$ and $\widetilde{\mathrm{A}}^{1} \mathrm{~A}^{\prime \prime}$ states of $\mathrm{HAsO}$ and $\mathrm{DAsO}$ evaluated using geometrical parameters of Table 2 (see footnotes of Table 2) and the experimental $\mathrm{r}_{0}$ values from reference 1.

\begin{tabular}{|c|c|c|c|}
\hline$\widetilde{\mathrm{X}}^{1} \mathrm{~A}^{\prime}$ & $\mathrm{A}$ & $\mathrm{B}$ & $\mathrm{C}$ \\
\hline $\operatorname{CCSD}\left(\mathrm{T}^{*}\right)-\mathrm{F} 12 / \mathrm{VQZ}-\mathrm{F} 12 \mathrm{r}_{\mathrm{e}}$ & 7.2830 & 0.4718 & 0.4431 \\
\hline MRCI-F12/VQZ-F12 $r_{e}$ & 7.2691 & 0.4712 & 0.4425 \\
\hline CCSD(T*)-F12/CVQZ-F12 $r_{e}$ & 7.3765 & 0.4773 & 0.4483 \\
\hline MRCI-F12/CVQZ-F12 $r_{e}$ & 7.4763 & 0.4767 & 0.4481 \\
\hline MRCI-F12/CVQZ-F12 PEF $r_{e}$ & 7.5088 & 0.4768 & 0.4483 \\
\hline MRCI-F12/CVQZ-F12 PEF $\mathrm{r}_{0}$ & 7.3618 & 0.4744 & 0.4457 \\
\hline Experimental $r_{0}$ from reference 1 & $7.271226(76)$ & $0.4742874(93)$ & $0.4442219(84)$ \\
\hline Derived $r_{e}(\text { combine theory and experiment })^{\mathrm{a}}$ & 7.3322 & 0.4761 & 0.4471 \\
\hline DAsO: MRCI-F12/CVQZ-F12 PEF $\mathrm{r}_{0}$ & 3.7889 & 0.4687 & 0.4171 \\
\hline DAsO: experimental $r_{0}$ & $3.737223(35)$ & $0.4688782(91)$ & $0.4153559(84)$ \\
\hline \multicolumn{4}{|l|}{$\widetilde{\mathrm{A}}^{1} \mathrm{~A}^{\prime \prime}$} \\
\hline MRCI-F12/VQZ-F12 $r_{\mathrm{e}}$ & 7.0812 & 0.4079 & 0.3873 \\
\hline MRCI-F12/CVQZ-F12 $r_{e}$ & 7.1969 & 0.4149 & 0.3923 \\
\hline MRCI-F12/CVQZ-F12 PEF $\mathrm{r}_{\mathrm{e}}$ & 7.2048 & 0.4152 & 0.3926 \\
\hline MRCI-F12/CVQZ-F12 PEF r 0 & 7.0255 & 0.4150 & 0.3919 \\
\hline Experimental $r_{0}$ from reference 1 & $7.06889(41)$ & $0.4161806(89)$ & $0.391788(87)$ \\
\hline Derived $r_{e}(\text { combine theory and experiment })^{a}$ & 7.0910 & 0.4157 & 0.3927 \\
\hline IFCA $r_{e}$ & 7.0759 & 0.4235 & 0.3996 \\
\hline IFCA $r_{0}$ & 6.9015 & 0.4234 & 0.3989 \\
\hline DAsO: MRCI-F12/CVQZ-F12 PEF $\mathrm{r}_{0}$ & 3.8553 & 0.4132 & 0.3705 \\
\hline DAsO: experimental $\mathrm{r}_{0}$ & $3.597941(26)$ & $0.4145662(86)$ & $0.3703298(85)$ \\
\hline
\end{tabular}




\begin{tabular}{|l|l|l|l|}
\hline DAsO: IFCA $\mathrm{r}_{0}$ & 3.5304 & 0.4209 & 0.3761 \\
\hline
\end{tabular}

${ }^{\mathrm{a}}$ The combined $r_{e}$ rotational constants were evaluated using the combined $r_{e}$ geometrical parameters, which were obtained by combining the experimental $r_{0}$ values from reference 1 and the differences between computed $r_{0}$ and $r_{e}$ values (from MRCI-F12/CVQZ-F12 PEF) (see footnote $d$ of Table 2 and text). 
Figure Captions

Figure 1. Simulated (top 4) and experimental (bottom from reference 1) $\widetilde{A}^{1} A^{\prime \prime}(0,0,0) \rightarrow \widetilde{X}^{1} A^{\prime} S V L$ emission spectra of HAsO.

Figure 2. Simulated (top 4) and experimental (bottom from reference 1) $\widetilde{A}^{1} A^{\prime \prime}(0,0,0) \rightarrow \widetilde{X}^{1} A^{\prime} S V L$ emission spectra of DAsO. 
Figure 1. Simulated (top 4) and experimental (bottom from reference 1) $\widetilde{A}^{1} A^{\prime \prime}(0,0,0) \rightarrow \widetilde{X}^{1} A^{\prime} S V L$ emission spectra of HAsO.

\section{Simulated A(0,0,0)-X SVL emission spectra of HAsO}

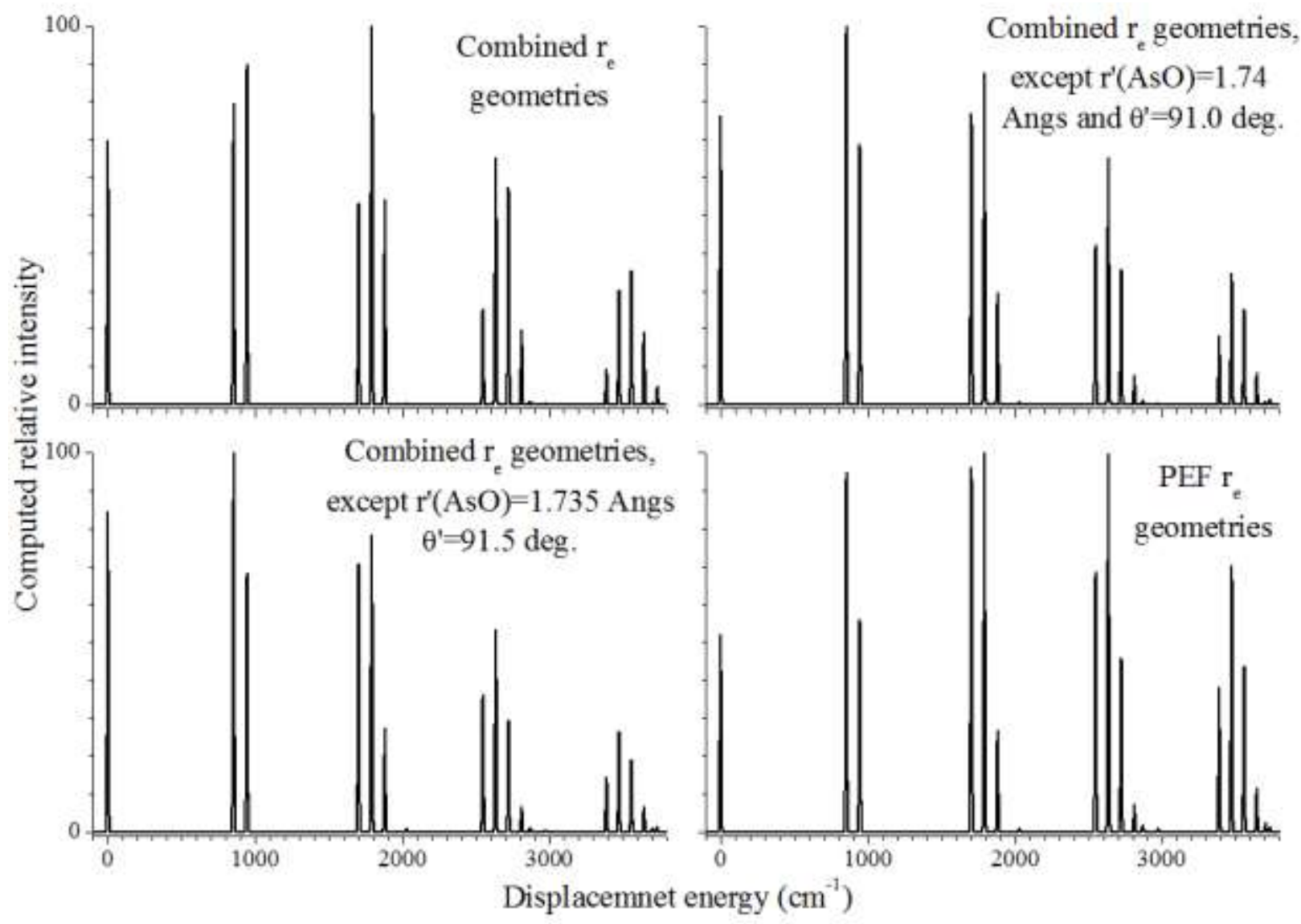

Experimental A(0,0,0)-X SVL emission of HAsO

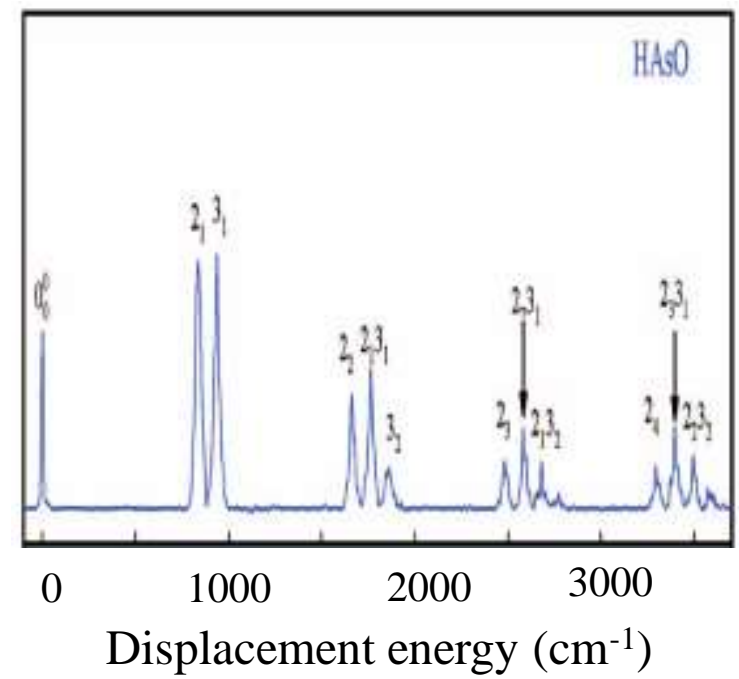


Figure 2. Simulated (top 4) and experimental (bottom from reference 1) $\widetilde{A}^{1} A^{\prime \prime}(0,0,0) \rightarrow \widetilde{X}^{1} A^{\prime} S V L$ emission spectra of DAsO.

\section{Simulated $\mathrm{A}(0,0,0)-\mathrm{X}$ SVL emission spectra of DAsO}

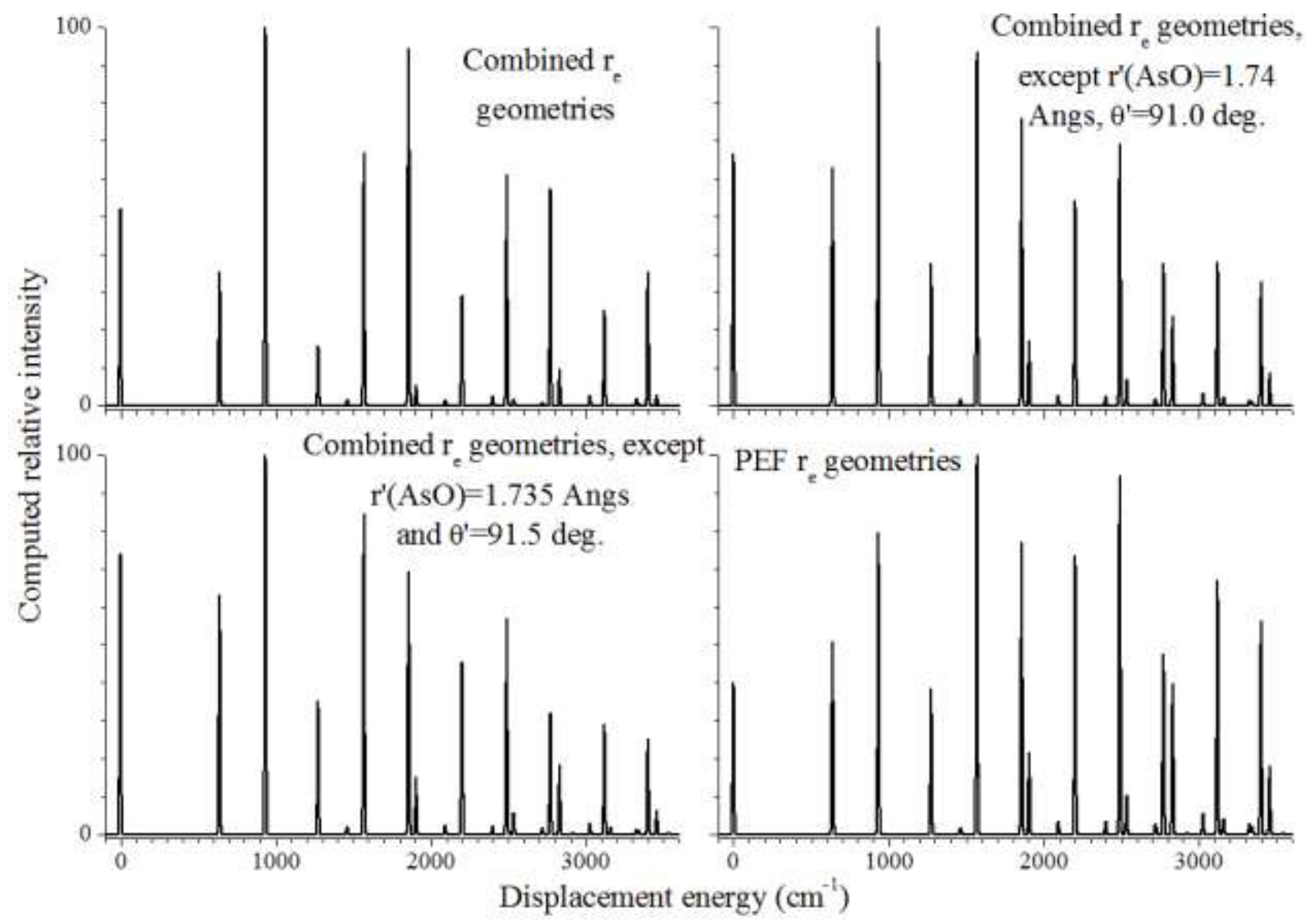

\section{Experimental $\mathrm{A}(0,0,0)-\mathrm{X}$ SVL emission of DAsO}

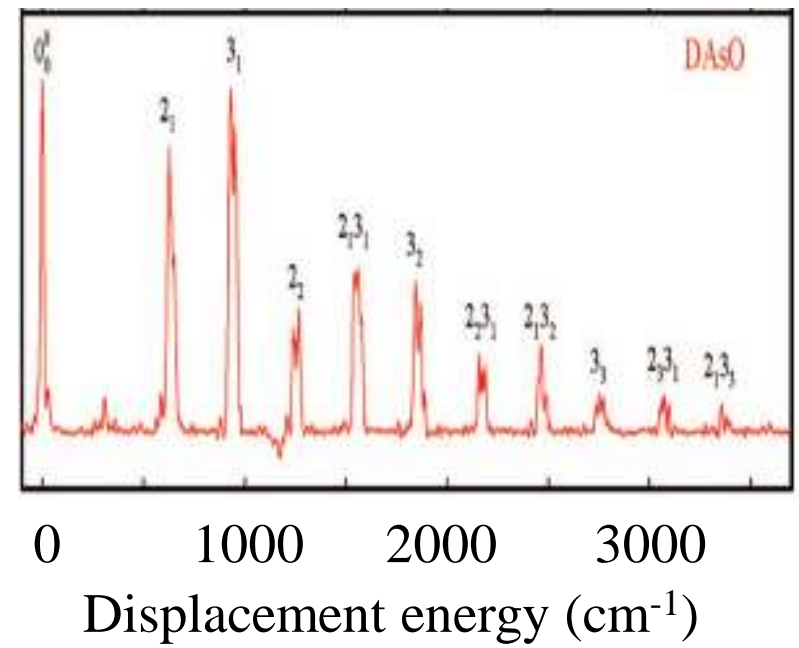




\section{References}

${ }^{1}$ R. Grimminger and D. J. Clouthier, J. Chem. Phys. 135, 184308 (2011).

${ }^{2}$ D. K. W. Mok, E. P. F. Lee, F.-T. Chau, D. C. Wang, and J. M. Dyke, J. Chem. Phys. 113, 5791 (2000).

${ }^{3}$ D. W. K. Mok, E. P. F. Lee, F.-T. Chau, and J. M. Dyke, J. Chem. Theor. Comput. 5, 565 (2009).

${ }^{4}$ D. K. W. Mok, E. P. F. Lee, F.-T. Chau, and J. M. Dyke, J. Comput. Chem. 22, 1896 (2001).

${ }^{5}$ F.-T. Chau, J. M. Dyke, E. P. F. Lee, and D. K. W. Mok, J. Chem. Phys. 115, 5816 (2001).

${ }^{6}$ E. P. F. Lee, D. K. W. Mok, J. M. Dyke, and F.-T. Chau, J. Phys. Chem. A 106, 10130 (2002).

${ }^{7}$ D. K. W. Mok, E. P. F. Lee, F.-T. Chau, and J. M. Dyke, J. Chem. Phys. 120, 1292 (2004).

${ }^{8}$ F.-T. Chau, D. K. W. Mok, E. P. F. Lee, and J. M. Dyke, J. Chem. Phys. 121, 1810 (2004).

${ }^{9}$ F.-T. Chau, D. K. W. Mok, E. P. F. Lee, and J. M. Dyke, ChemPhysChem 6, 2037 (2005).

${ }^{10}$ J. M. Dyke, E. P. F. Lee, D. K. W. Mok, and F.-T. Chau, ChemPhysChem 6, 2046 (2005).

${ }^{11}$ D. K. W. Mok, F.-T. Chau, E. P. F. Lee, and J. M. Dyke, J. Comput. Chem. 31, 476 (2010).

${ }^{12}$ E. P. F. Lee, D. K. W. Mok, F.-T. Chau, and J. M. Dyke, J. Chem. Phys. 132, 234309 (2010).

${ }^{13}$ E. P. F. Lee, D. K. W. Mok, F.-T. Chau, and J. M. Dyke, J. Chem. Phys. 127, 214305 (2007).

${ }^{14}$ D. K. W. Mok, E. P. F. Lee, F.-T. Chau, and J. M. Dyke, J. Chem. Phys. 140, 194311 (2014).

${ }^{15}$ W. Schneider, W. Thiel, and A. Komornicki, J. Phys. Chem. 94, 2810 (1990).

${ }^{16}$ L. Andrews, R. Withnall, and B. W. Moores, J. Phys. Chem. 93, 1279 (1989).

${ }^{17}$ H.-J. Werner, P. J. Knowles, G. Knizia, F. R. Manby, M. Schütz, P. Celani, T. Korona, R. Lindh, A. Mitrushenkov, G. Rauhut, K. R. Shamasundar, T. B. Adler, R. D. Amos, A. Bernhardsson, A. Berning, D. L. Cooper, M. J. O. Deegan, A. J. Dobbyn, F. Eckert, E. Goll, C. Hampel, A. Hesselmann, G. Hetzer, T. Hrenar, G. Jansen, C. Köppl, Y. Liu, A. W. Lloyd, R. A. Mata, A. J. May, S. J. McNicholas, W. Meyer, M. E. Mura, A. Nicklass, D. P. O'Neill, P. Palmieri, D. Peng, K. Pflüger, R. Pitzer, M. Reiher, T. Shiozaki, H. Stoll, A. J. Stone, R. Tarroni, T. Thorsteinsson, and M. Wang, MOLPRO, version 2012.1, a package of ab initio programs, see http://www.molpro.net.

${ }^{18}$ H.-J. Werner, P. J. Knowles, G. Knizia, F. R. Manby and M. Schütz, WIREs Comput. Mol. Sci. 2, 242-253 (2012).

${ }^{19}$ H.-J. Werner and P. J. Knowles, J. Chem. Phys. 82, 5053 (1985).

${ }^{20}$ C. Angeli, M. Pastore and R. Cimiraglia, Theor. Chem. Acc., 117, 743 (2007).

${ }^{21}$ T. Shiozaki and H.-J. Werner, J. Chem. Phys. 133, 141103 (2010).

${ }^{22}$ T. Shiozaki, G. Knizia, and H.-J. Werner, J. Chem. Phys. 134, 034113 (2011).

${ }^{23}$ G. Knizia, T. B. Adler, and H.-J. Werner, J. Chem. Phys. 130, 054104 (2009).

${ }^{24}$ C. D. Sherrill, J. Chem. Phys. 132, 110902 (2010).

${ }^{25}$ T. B. Adler, G. Knizia and H.-J. Werner, J. Chem. Phys. 127, 221106 (2007). 
${ }^{26}$ G. Hill and K. A. Peterson, J. Chem. Phys. 141, 094106 (2014).

${ }^{27}$ F. Weigend: J. Comput. Chem. 29, 167 (2008).

${ }^{28}$ K. E. Yousaf and K. A. Peterson, J. Chem. Phys. 129, 184108 (2008).

${ }^{29}$ Christof Hattig, auxiliary basis sets for RI-MP2 calculations, TURBOMOLE basis set library 6.0, 1 (2009).

${ }^{30}$ D. Feller, EMSL Basis Set Library: J. Comp. Chem., 17(13), 1571-1586 (1996).

${ }^{31}$ K.L. Schuchardt, B.T. Didier, T. Elsethagen, L. Sun, V. Gurumoorthi, J. Chase, J. Li and T.L. Windus, Basis Set Exchange: A Community Database for Computational Sciences: J. Chem. Inf. Model., 47(3), 1045-1052, (2007), doi:10.1021/ci600510j.

${ }^{32}$ K.A. Peterson, T. Adler, and H.-J. Werner, J. Chem. Phys. 128, 084102 (2008).

33 T.H. Dunning, Jr. J. Chem. Phys. 90, 1007 (1989).

${ }^{34}$ K.A. Peterson, J. Chem. Phys. 119, 11099 (2003).

35 J. G. Hill, S. Mazumder and K. A. Peterson, J. Chem. Phys. 132, 054108 (2010).

${ }^{36}$ G. Herzberg, Molecular Spectra and Molecular Structure III. Electronic Spectra and Electronic Structure of Polyatomic Molecules (Van Nostrand, New York, 1966), p. 142.

${ }^{37}$ Photomultiplier Handbook, information furnished by BURLE INDUSTRIES, INC.; from http://psec.uchicago.edu/links/Photomultiplier_Handbook.pdf http://science.unitn.it/ semicon/members/pavesi/c31034.pdf (accessed 21-1-2016). 